\title{
Some Inequalities of the Hermite-Hadamard Type for Harmonically Quasi-Convex Functions
}

\author{
Chun-Long Li ${ }^{1, *}$, Gui-Hua Gu${ }^{1}$, Bai-Ni Guo ${ }^{2}$ \\ ${ }^{1}$ College of Mathematics, Inner Mongolia University for Nationalities, Tongliao, Inner Mongolia, China \\ ${ }^{2}$ School of Mathematics and Informatics, Henan Polytechnic University, Jiaozuo, Henan, 454010, China \\ *Corresponding author: lichunlong70@163.com
}

Received October 31, 2017; Revised December 01, 2017; Accepted December 06, 2017

\begin{abstract}
In the paper, by Holder's integral inequality, the authors establish some Hermite-Hadamard type integral inequalities for harmonically quasi-convex functions.
\end{abstract}

Keywords: Hermite-Hadamard inequality, harmonically quasi-convex function, Holder's integral inequality

Cite This Article: Chun-Long Li, Gui-Hua Gu, and Bai-Ni Guo, "Some Inequalities of the HermiteHadamard Type for Harmonically Quasi-Convex Functions.” Turkish Journal of Analysis and Number Theory, vol. 5, no. 6 (2017): 226-229. doi: 10.12691/tjant-5-6-4.

\section{Introduction}

The following definitions for various convex functions are well known in the literature.

Definition 1.1 A function $f: I \subseteq R \rightarrow R$ is said to be convex if

$$
f(\lambda x+(1-\lambda) y) \leq \lambda f(x)+(1-\lambda) f(y)
$$

holds for all $x, y \in I$ and $\lambda \in[0,1]$.

Definition $1.2([1,2,3])$ A function $f: I \subseteq R \rightarrow R$ is said to be quasi-convex if

$$
f(\lambda x+(1-\lambda) y) \leq \max \{f(x), f(y)\}
$$

holds for all $x, y \in I$ and $\lambda \in[0,1]$.

Definition 1.3 ([4]) For $f:[0, b] \rightarrow R$ with $b>0$ and $m \in(0,1]$, if

$$
f(\lambda x+m(1-\lambda) y) \leq \lambda f(x)+m(1-\lambda) f(y)
$$

is valid for all $x, y \in[0, b]$ and $\lambda \in[0,1]$, then we say that $f(x)$ is an $m$-convex function on $[0, b]$.

Definition 1.4 ([5]) Let $f:[0, b] \rightarrow R$ with $b>0$ and $(\alpha, m) \in(0,1]^{2}$. If

$$
f(\lambda x+m(1-\lambda) y) \leq \lambda^{\alpha} f(x)+m\left(1-\lambda^{\alpha}\right) f(y)
$$

is valid for all $x, y \in[0, b]$ and $\lambda \in[0,1]$, then we say that $f(x)$ is an $(\alpha, m)$-convex function on $[0, b]$.

Definition 1.5 ([9]) A function $f: I \subseteq R_{+}=(0, \infty) \rightarrow R$ is said to be a harmonically quasi-convex function on $I$ if

$$
f\left(\left(\frac{t}{x}+\frac{1-t}{y}\right)^{-1}\right) \leq \max \{f(x), f(y)\}
$$

holds for all $x, y \in I$ and $t \in[0,1]$.

In $[7,8]$, the following inequalities of HermiteHadamard type were established.

Theorem 1.1 ([7], Theorems 2.2 and 2.3]). Let $f: I^{\circ} \subseteq R \rightarrow R$ be a differentiable mapping and $a, b \in I^{\circ}$ with $a<b$. Then

(i) if $\left|f^{\prime}\right|$ is convex on $[a, b]$, then

$\left|\frac{f(a)+f(b)}{2}-\frac{1}{b-a} \int_{a}^{b} f(x) \mathrm{d} x\right| \leq \frac{(b-a)\left(\left|f^{\prime}(a)\right|+\left|f^{\prime}(b)\right|\right)}{8} ;$

(ii) if $\left|f^{\prime}\right|^{p /(p-1)}$ is convex on $[a, b]$ for $p>1$, then

$$
\begin{aligned}
& \left|\frac{f(a)+f(b)}{2}-\frac{1}{b-a} \int_{a}^{b} f(x) \mathrm{d} x\right| \\
& \leq \frac{b-a}{2(p+1)^{1 / p}}\left(\frac{\left|f^{\prime}(a)\right|^{p /(p-1)}+\left|f^{\prime}(b)\right|^{p /(p-1)}}{2}\right)^{(p-1) / p} .
\end{aligned}
$$

Theorem 1.2 ([8], Theorem 2.3]) Let $f: I \subseteq R \rightarrow R$ be differentiable on $I^{\circ}$ and $a, b \in I$ with $a<b$. If $\left|f^{\prime}\right|^{p}$ is s-convex on $[a, b]$ for $p>1$, then

$$
\begin{aligned}
& \left|f\left(\frac{a+b}{2}\right)-\frac{1}{b-a} \int_{a}^{b} f(x) \mathrm{d} x\right| \\
& \leq \frac{b-a}{4}\left(\frac{4}{p+1}\right)^{1 / p}\left(\left|f^{\prime}(a)\right|+\left|f^{\prime}(b)\right|\right) .
\end{aligned}
$$


In this paper, we will create some integral inequalities of Hermite-Hadamard type for harmonically quasi-convex functions.

\section{A Lemma}

For creating some integral inequalities of HermiteHadamard type for harmonically quasi-convex functions, we need the following lemma.

Lemma 2.1 Let $f: I \subseteq R_{+} \rightarrow R$ be differentiable on $I$, $a, b \in I$ with $a<b$, and $\theta \in(0,1]$. If $f^{\prime} \in L_{1}([a, b])$, then

$$
\begin{aligned}
& \frac{H(\theta, a, b)-a}{2 a H(\theta, a, b)} \int_{0}^{1}\left[\begin{array}{l}
(1-2 t)\left(\frac{t}{a}+\frac{1-t}{H(\theta, a, b)}\right)^{-2} \\
f^{\prime}\left(\left(\frac{t}{a}+\frac{1-t}{H(\theta, a, b)}\right)^{-1}\right)
\end{array}\right] \mathrm{d} t \\
& =\frac{f(a)+f(H(\theta, a, b))}{2} \\
& -\frac{a H(\theta, a, b)}{H(\theta, a, b)-a} \int_{a}^{H(\theta, a, b)} \frac{f(x)}{x^{2}} \mathrm{~d} x,
\end{aligned}
$$

where $H(\theta, a, b)=\frac{a b}{\theta a+(1-\theta) b}$.

Proof Integrating by part and changing variables $x=\left(t a^{-1}+(1-t)[H(\theta, a, b)]^{-1}\right)^{-1}$ for $t \in[0,1]$ yield

$$
\begin{aligned}
& \int_{0}^{1}(1-2 t)\left(\frac{t}{a}+\frac{1-t}{H(\theta, a, b)}\right)^{-2} f^{\prime}\left(\left(\frac{t}{a}+\frac{1-t}{H(\theta, a, b)}\right)^{-1}\right) \mathrm{d} t \\
= & \frac{a H(\theta, a, b)}{H(\theta, a, b)-a}(f(a)+f(H(\theta, a, b))) \\
- & \frac{2[a H(\theta, a, b)]^{2}}{[H(\theta, a, b)-a]^{2}} f^{\prime}\left(\left(\frac{t}{a}+\frac{1-t}{H(\theta, a, b)}\right)^{-1}\right) \mathrm{d} t \\
= & \frac{a H(\theta, a, b)}{H(\theta, a, b)-a}(f(a)+f(H(\theta, a, b))) \\
- & \frac{2[a H(\theta, a, b)]^{2}}{[H(\theta, a, b)-a]^{2}} \int_{a}^{H(\theta, a, b)} \frac{f(x)}{x^{2}} \mathrm{~d} x .
\end{aligned}
$$

Lemma 2.1 is thus proved.

\section{Some New Integral Inequalities of Hermite-Hadamard Type}

Now we set off to create some integral inequalities of Hermite-Hadamard type for harmonically quasi-convex functions.

Theorem 3.1 Let $f: I \subseteq R_{+} \rightarrow R$ be a differentiable function, $a, b \in I$ with $a<b, \quad \theta \in(0,1]$, and $\left|f^{\prime}\right| \in L_{1}([a, b])$. If $\left|f^{\prime}\right|$ is harmonically quasi-convex on $[a, b]$, then

$$
\left|\begin{array}{l}
\frac{f(a)+f(H(\theta, a, b))}{2} \\
-\frac{a H(\theta, a, b)}{H(\theta, a, b)-a} \int_{a}^{H(\theta, a, b)} \frac{f(x)}{x^{2}} \mathrm{~d} x
\end{array}\right|
$$

$$
\begin{aligned}
& \leq \frac{\left\{\begin{array}{l}
{[H(\theta, a, b)-a]^{2}} \\
\left.+2 a H(\theta, a, b)\left(\begin{array}{l}
2 \ln H\left(\frac{1}{2}, a, H(\theta, a, b)\right) \\
-\ln [a H(a, b)]
\end{array}\right)\right\}
\end{array}\right.}{2[H(\theta, a, b)-a]} \\
& \quad \times \max \left\{\left|f^{\prime}(a)\right|,\left|f^{\prime}(H(\theta, a, b))\right|\right\} .
\end{aligned}
$$

Proof Using Lemma 2.1 and the harmonic quasi-convexity of $\left|f^{\prime}\right|$, we have

$$
\begin{aligned}
& \left|\begin{array}{l}
\frac{f(a)+f(H(\theta, a, b))}{2} \\
-\frac{a H(\theta, a, b)}{H(\theta, a, b)-a} \int_{a}^{H(\theta, a, b)} \frac{f(x)}{x^{2}} \mathrm{~d} x
\end{array}\right| \\
& \leq \frac{H(\theta, a, b)-a}{2 a H(\theta, a, b)} \int_{0}^{1}\left[\begin{array}{l}
|1-2 t|\left(\frac{t}{a}+\frac{1-t}{H(\theta, a, b)}\right)^{-2} \\
f^{\prime}\left(\left(\frac{t}{a}+\frac{1-t}{H(\theta, a, b)}\right)^{-1}\right) \mid
\end{array}\right] d t \\
& \leq \frac{H(\theta, a, b)-a}{2 a H(\theta, a, b)} \max \left\{\left|f^{\prime}(a)\right|,\left|f^{\prime}(H(\theta, a, b))\right|\right\} \\
& \times \int_{0}^{1}|1-2 t|\left(\frac{t}{a}+\frac{1-t}{H(\theta, a, b)}\right)^{-2} \mathrm{~d} t \\
& =\frac{\left\{\begin{array}{l}
{[H(\theta, a, b)-a]^{2}} \\
\left.+2 a H(\theta, a, b)\left(\begin{array}{l}
2 \ln H\left(\frac{1}{2}, a, H(\theta, a, b)\right) \\
-\ln [a H(a, b)]
\end{array}\right)\right\}
\end{array}\right.}{2[H(\theta, a, b)-a]} \\
& \times \max \left\{\left|f^{\prime}(a)\right|,\left|f^{\prime}(H(\theta, a, b))\right|\right\} .
\end{aligned}
$$

The proof of Theorem 3.1 is complete.

Theorem 3.2 Let $f: I \subseteq R_{+} \rightarrow R$ be a differentiable function, $a, b \in I$ with $a<b, \quad \theta \in(0,1]$, and $\left|f^{\prime}\right| \in L_{1}((0, b])$. If $\left|f^{\prime}\right|^{q}$ is harmonically quasi-convex on $[a, b]$ and $q>1$, then

$$
\begin{aligned}
& \left|\begin{array}{l}
\frac{f(a)+f(H(\theta, a, b))}{2} \\
-\frac{a H(\theta, a, b)}{H(\theta, a, b)-a} \int_{a}^{H(\theta, a, b)} \frac{f(x)}{x^{2}} \mathrm{~d} x
\end{array}\right| \\
& \leq \frac{\left[\begin{array}{l}
((q-1)[H(\theta, a, b)-a])^{1-1 / q} \\
\times\left([H(\theta, a, b)]^{2 q-1}-a^{2 q-1}\right)^{1 / q}
\end{array}\right]}{2[(q-1) a H(\theta, a, b)]^{1-1 / q}} \\
& \max \left\{\left|f^{\prime}(a)\right|,\left|f^{\prime}(H(\theta, a, b))\right|\right\} .
\end{aligned}
$$


Proof Since $\left|f^{\prime}\right|^{q}$ is harmonically quasi-convex on $[a, b]$, by Lemma 2.1 and Hölder's inequality, we have

$$
\begin{aligned}
& \left|\frac{f(a)+f(H(\theta, a, b))}{2}-\frac{a H(\theta, a, b)}{H(\theta, a, b)-a} \int_{a}^{H(\theta, a, b)} \frac{f(x)}{x^{2}} \mathrm{~d} x\right| \\
& \leq\left.\frac{H(\theta, a, b)-a}{2 a H(\theta, a, b)}\left|\int_{0}^{1}\right|\left(\frac{t}{a}+\frac{1-t}{H(\theta, a, b)}\right)^{-2 q}\left(\left(\frac{t}{a}+\frac{1-t}{H(\theta, a, b)}\right)^{-1}\right)\right|^{q} \mathrm{~d} t \mid \\
& \quad \times\left(\int_{0}^{1}|1-2 t|^{q /(q-1)} \mathrm{d} t\right)^{1-1 / q} \\
& \leq \frac{H(\theta, a, b)-a}{2 a H(\theta, a, b)}\left(\int_{0}^{1}\left(\frac{t}{a}+\frac{1-t}{H(\theta, a, b)}\right)^{-2 q} \mathrm{~d} t\right)^{1 / q} \\
& \quad \times\left(\int_{0}^{1}|1-2 t|^{q /(q-1)} \mathrm{d} t\right)^{1-1 / q} \\
& \quad \times \max \left\{\left|f^{\prime}(a)\right|,\left|f^{\prime}(H(\theta, a, b))\right|\right\} \\
& =\frac{\left[\begin{array}{l}
((q-1)[H(\theta, a, b)-a])^{1-1 / q} \\
\times(
\end{array}\right]}{\left.\left.2[H(\theta, a, b)]^{2 q-1}-a^{2 q-1}\right)^{1 / q}\right]} \\
& \times \max \left\{\left|f^{\prime}(a)\right|,\left|f^{\prime}(H(\theta, a, b))\right|\right\} .
\end{aligned}
$$

Theorem 3.2 is thus proved.

Theorem 3.3. Let $f: I \subseteq R_{+} \rightarrow R$ be a differentiable function, $a, b \in I$ with $a<b, \quad \theta \in(0,1]$, and $\left|f^{\prime}\right| \in L_{1}((0, b])$. If $\left|f^{\prime}\right|^{q}$ is harmonically quasi-convex on $[a, b]$ for $q>1$, then

$$
\begin{aligned}
& \mid \begin{array}{l}
\mid \frac{f(a)+f(H(\theta, a, b))}{2} \\
-\frac{a H(\theta, a, b)}{H(\theta, a, b)-a} \int_{a}^{H(\theta, a, b)} \frac{f(x)}{x^{2}} \mathrm{~d} x \mid
\end{array} \\
& \leq \frac{\{(H(\theta, a, b)-a]}{\left.\left(a^{2 q /(q-1)}+[H(\theta, a, b)]^{2 q /(q-1)}\right)^{1-1 / q}\right\}} \\
& 2^{(3 q-1) / q} a H(\theta, a, b) \\
& \max \left\{\left|f^{\prime}(a)\right|,\left|f^{\prime}(H(\theta, a, b))\right|\right\} .
\end{aligned}
$$

Proof From the GA-inequality, we have

$$
\begin{aligned}
& \left(\frac{t}{a}+\frac{1-t}{H(\theta, a, b)}\right)^{-2 q /(q-1)} \\
& \leq t a^{2 q /(q-1)}+(1-t)[H(\theta, a, b)]^{2 q /(q-1)}
\end{aligned}
$$

for all $t \in[0,1]$. By Lemma 2.1 and the harmonic quasi-convexity of $\left|f^{\prime}\right|^{q}$ and Hölder's inequality, we have

$$
\left|\begin{array}{l}
\frac{f(a)+f(H(\theta, a, b))}{2} \\
-\frac{a H(\theta, a, b)}{H(\theta, a, b)-a} \int_{a}^{H(\theta, a, b)} \frac{f(x)}{x^{2}} \mathrm{~d} x
\end{array}\right|
$$$$
\leq \frac{H(\theta, a, b)-a}{2 a H(\theta, a, b)}
$$$$
\begin{aligned}
& \times\left(\int_{0}^{1}|1-2 t|\left|f^{\prime}\left(\left(\frac{t}{a}+\frac{1-t}{H(\theta, a, b)}\right)^{-1}\right)\right|^{q} \mathrm{~d} t\right)^{1 / q} \\
& \times\left(\int_{0}^{1}|1-2 t|\left(\frac{t}{a}+\frac{1-t}{H(\theta, a, b)}\right)^{-2 q /(q-1)} \mathrm{d} t\right)^{1-1 / q}
\end{aligned}
$$$$
\leq \frac{H(\theta, a, b)-a}{2 a H(\theta, a, b)}\left(\int_{0}^{1}|1-2 t| \max \left\{\begin{array}{l}
\left|f^{\prime}(a)\right|^{q}, \\
\left|f^{\prime}(H(\theta, a, b))\right|^{q}
\end{array}\right\} \mathrm{d} t\right)^{1 / q}
$$$$
\times\left(\int_{0}^{1}|1-2 t|\left(\frac{t}{a}+\frac{1-t}{H(\theta, a, b)}\right)^{-2 q /(q-1)} \mathrm{d} t\right)^{1-1 / q}
$$$$
=\frac{\left\{\begin{array}{l}
{[H(\theta, a, b)-a]} \\
\times\left(a^{2 q /(q-1)}+[H(\theta, a, b)]^{2 q /(q-1)}\right)^{1-1 / q}
\end{array}\right\}}{2^{(3 q-1) / q} a H(\theta, a, b)}
$$$$
\max \left\{\left|f^{\prime}(a)\right|,\left|f^{\prime}(H(\theta, a, b))\right|\right\} .
$$

The proof of Theorem 3.3 is complete.

\section{References}

[1] W. Fenchel, Convex cones, sets, and functions, Mimeographed Lectures Notes, Princeton University, Princeton, New Jersey, 1951.

[2] K. L. Arrow and C. Enthovena, Quasi-concave programming, Econometrica, 1961, 29: 779-800.

[3] S. S. Dragomir, J. Pečaric and L. E. Persson, Some inequalities of Hadamard type, Soochow J. Math. 21 (1995), no. 3, 335-341.

[4] G. Toader. Some generalizations of the convexity. Proceedings of the Colloquium on Approximation and Optimization, Univ.ClujNapoca, Cluj-Napoca, 1985.

[5] V. G. Miheşan, A generalization of the convexity, Seminar on Functional Equations, Approx. and Convex., Cluj-Napoca (Romania), 1993.

[6] Bo-Yan Xi, Tian-Yu Zhang, and Feng Qi. Some inequalities of Hermite-Hadamard type for $m$-harmonic-arithmetically convex functions. ScienceAsia, 2015, 41 (5): 357-361.

[7] S. S. Dragomir and R. P. Agarwal, Two inequalities for differentiable mappings and applications to special means of real numbers and to trapezoidal formula, Appl. Math. Lett., 1998, 11: 91-95.

[8] U. S. Kirmaci, Inequalities for differentiable mappings and applications to special means of real numbers and to midpoint formula, Appl. Math. Comp., 147 (2004), 137-146.

[9] Tian-Yu Zhang, Ai-Ping Ji, and Feng Qi. Integral inequalities of Hermite-Hadamard type for harmonically quasi-convex functions. Proceedings of the Jangjeon Mathematical Society, 2013, 16 (3), 399-407.

[10] S. S. Dragomir, On some new inequalities of Hermite-Hadamard type for $m$-convex functions, Tamkang J. Math. 33 (2002) 45-55. 
[11] S. S. Dragomir, G. Toader, Some inequalities for $m$-convex functions, Studia Univ. Babẹs-Bolyai Math. 38 (1993) 21-28.

[12] Bo-Yan Xi and Feng Qi. Some new integral inequalities of Hermite-Hadamard type for (log, $(\alpha, m)$ )-convex functions on coordinates. Studia Universitatis Babęs-Bolyai Mathematica, 2015, 60 (4): 509-525.

[13] Bo-Yan Xi and Feng Qi. Integral inequalities of HermiteHadamard type for $((\alpha, m), \log )$-convex functions on co-ordinates. Problemy Analiza-Issues of Analysis, 2015, 22 (2): 73-92.

[14] Bo-Yan Xi and Feng Qi. Hermite-Hadamard type inequalities for geometrically $r$-convex functions. Studia Scientiarum Mathematicarum Hungarica, 2014, 51(4): 530-546.
[15] Bo-Yan Xi and Feng Qi. Some Hermite-Hadamard type inequalities for differentiable convex functions and applications. Hacettepe Journal of Mathematics and Statistics, 2013, 42(3): 243-257.

[16] Bo-Yan Xi and Feng Qi. Integral inequalities of Simpson type for logarithmically convex functions. Advanced Studies in Contemporary Mathematics, 2013, 23(4): 559-566.

[17] Bo-Yan Xi and Feng Qi. Hermite-Hadamard type inequalities for functions whose derivatives are of convexities. Nonlinear Functional Analysis and Applications, 2013, 18(2),: 163-176. 